\title{
Genome-wide in silico identification of membrane-bound transcription factors in plant species
}

\author{
Shixiang Yao ${ }^{1}$, Lili Deng ${ }^{1}$, Kaifang Zeng ${ }^{\text {Corresp. } 1}$ \\ ${ }^{1}$ College of Food Science, Southwest University, Chongqing, China \\ Corresponding Author: Kaifang Zeng \\ Email address: zengkaifang@hotmail.com
}

Membrane-bound transcription factors (MTFs) are located in cellular membranes due to their transmembrane domains. In plants, proteolytic processing is considered to be the main mechanism for MTF activation, which ensures the liberation of MTFs from membranes and further their translocation into the nucleus to regulate gene expression; this process skips both the transcriptional and translational stages, and thus it guarantees the prompt responses of plants to various stimuli. Currently, information concerning plant MTFs is limited to model organisms, including Arabidopsis thaliana and Oryza sativa, and little is known in other plant species at the genome level. In the present study, seven membrane topology predictors widely used by the research community were employed to establish a reliable workflow for MTF identification. Genome-wide in silico analysis of MTFs was then performed in 14 plant species spanning the chlorophytes, bryophytes, gymnosperms, monocots and eudicots. A total of 1,089 MTFs have been identified from a total of 25,850 transcription factors in these 14 plant species. These MTFs belong to 52 gene family, and the top six most abundant families are the NAC (128), SBP (77), C2H2 (70), bZIP (67), MYBrelated (65) and bHLH (63) families. The MTFs have transmembrane spans ranging from one to thirteen, and $71.5 \%$ and $21.1 \%$ of the MTFs have one and two transmembrane motifs, respectively. Most of the MTFs in this study have transmembrane motifs located in either $\mathrm{N}$ - or $\mathrm{C}$-terminal regions, indicating that proteolytic cleavage could be a conserved mechanism for MTF activation. Additionally, approximately half of the MTFs in the genome of either Arabidopsis thaliana or Gossypium raimondii could be potentially regulated by alternative splicing, indicating that alternative splicing is another conserved activation mechanism for MTFs. The present study performed systematic analyses of MTFs in plant lineages at the genome level, and provides invaluable information for the research community. 


\section{Manuscript to be reviewed}

1 Genome-wide in silico identification of membrane-bound transcription

2

4

5

6

7

8

9

10

11

12

13

14

15

16

17

18

19

20

21

22

23

24

25

26

27

28

29

30

31

factors in plant species

Shixiang Yao, Lili Deng and Kaifang Zeng

College of Food Science, Southwest University, Chongqing 400715, China;

Author for correspondence:

Kaifang Zeng

Tel: +861068251298

Email: zkaifang@swu.edu.cn; zengkaifang@hotmail.com

\section{Abstract}

Membrane-bound transcription factors (MTFs) are located in cellular membranes due to their transmembrane domains. In plants, proteolytic processing is considered to be the main mechanism for MTF activation, which ensures the liberation of MTFs from membranes and further their translocation into the nucleus to regulate gene expression; this process skips both the transcriptional and translational stages, and thus it guarantees the prompt responses of plants to various stimuli. Currently, information concerning plant MTFs is limited to model organisms, including Arabidopsis thaliana and Oryza sativa, and little is known in other plant species at the genome level. In the present study, seven membrane topology predictors widely used by the research community were employed to establish a reliable workflow for MTF identification. Genome-wide in silico analysis of MTFs was then performed in 14 plant species spanning the chlorophytes, bryophytes, gymnosperms, monocots and eudicots. A total of 1,089 MTFs have been identified from a total of 25,850 transcription factors in these 14 plant species. These MTFs belong to 52 gene family, and the top six most abundant families are the NAC (128), SBP (77), C2H2 (70), bZIP (67), MYB-related (65) and bHLH (63) families. The MTFs have transmembrane spans ranging from one to thirteen, and $71.5 \%$ and $21.1 \%$ of the MTFs have one and two transmembrane motifs, respectively. Most of the MTFs in this study have transmembrane motifs located in either $\mathrm{N}$ - or $\mathrm{C}$-terminal regions, indicating that proteolytic cleavage could be a conserved mechanism for MTF activation. Additionally, approximately half of the MTFs in the genome of either Arabidopsis thaliana or 
Gossypium raimondii could be potentially regulated by alternative splicing, indicating that alternative splicing is another conserved activation mechanism for MTFs. The present study performed systematic analyses of MTFs in plant lineages at the genome level, and provides invaluable information for the research community.

\section{Introduction}

Transcription factors play a primary regulatory role in gene transcription and thus ensure normal growth of plants and promote their adaptation to environmental stress ( $\mathrm{Li}$ et al. 2011). Transcription factors are tightly regulated at multiple levels, including the transcriptional, translational, and post-translational levels (Seo et al. 2008). During the last decade, a novel mechanism of post-translational regulation of transcription factors-proteolytic processing dependent activation-has been extensively studied and well established (Seo 2014). A small proportion of transcription factors containing transmembrane (TM) motifs are translated in the cytoplasm and then rapidly anchored to cellular membranes including plasma, mitochondrial, endoplasmic reticulum (ER) membranes, rather than being translocated to the nucleus (Kim et al. 2010). Membrane-bound transcription factors (MTFs) are stored in dormant forms and become active after being released from membrane. Most MTFs with known functions have a single TM domain located adjacent to their transcription factor domains located within either the $\mathrm{N}$-terminal or $\mathrm{C}$-terminal region and they are anchored to the membrane by TM motifs. Thus, in response to environmental stimuli and physiological signals, the MTFs can be cleaved near the TM region by a specific protease, and the transcription factor can be released from the membrane and then translocated into the nucleus to exert its functions (Che et al. 2010). The proteolytic processing-dependent activation of MTFs skips both transcriptional and translational steps, and it thus ensures the prompt response of plants to exogenous and endogenous signals.

Since the first plant MTF, AtbZIP60, was elucidated at the molecular level, a major focus of research on the MTFs of plants has targeted NAC and bZIP transcription factors (Iwata and Koizumi 2005; Seo 2014). For instance, the function of several MTFs has been 


\section{Manuscript to be reviewed}

61 well elucidated in recent years, including eight NAC and three bZIP members (Seo 2014).

62 Previous studies of plant MTFs suggested that MTFs were involved in various aspects of 63 plant growth, development and environmental responses, such as seed germination (Park 64 et al. 2011), cell division (Kim et al. 2006), root hair development (Slabaugh et al. 2011), 65 sugar signaling ( $\mathrm{Li}$ et al. 2011), ER stress (Che et al. 2010; Iwata et al. 2008; Iwata and 66 Koizumi 2005; Yang et al. 2014), reactive oxygen species (ROS) signaling (De Clercq et al. 2013; Lee et al. 2012; $\mathrm{Ng}$ et al. 2013), cold stress (Seo et al. 2010), heat stress (Gao et al. 2008), salt stress (Che et al. 2010; Kim et al. 2008) and osmotic stress (Kim et al. 2012; Yoon et al. 2008). A further genome wide analysis of MTFs in Arabidopsis (Arabidopsis thaliana) and rice (Oryza sativa) showed that many transcription factor families contained membrane-bound members, although most of them have yet to be functionally resolved (Kim et al. 2010). MTFs are fast emerging as critical drivers of gene regulation in response to various stimuli in plants. Because of their crucial role in the regulation of gene expression, it is of great importance to identify MTFs in other plant species. However, most of the MTFs that have been functionally resolved are those from Arabidopsis. This may be partially because the genome-wide analyses of MTFs have been performed using genomes of model organisms including Arabidopsis and rice. Thus, it is of great importance that a systematic analysis of MTFs is performed in various plant lineages besides Arabidopsis and rice at the genome level to not only widen the knowledge of MTFs in plant species but also provide invaluable resources for the community and further stimulate the functional elucidation of these transcription factors.

Here, we established a workflow for the identification of plant MTFs and performed genome-wide analyses of MTFs in 14 plant species spanning the chlorophytes, bryophytes, gymnosperms, monocots and eudicots. A total of 1089 MTFs belonging to 52 gene families were identified in these plant species. Most of the MTFs have one or two transmembrane motifs located in either the $\mathrm{N}$ - or C-terminal region, indicating that proteolytic cleavage could be a conserved mechanism for MTF activation. Additionally, a considerable proportion of the MTFs in the Arabidopsis and cotton (Gossypium raimondii) 
89 genome were expected to be activated by alternative splicing (ALS), indicating that ALS is

90 another conserve regulatory mechanism for the activation of MTFs.

\section{Materials \& Methods}

\section{Sequence retrieval for whole membrane-bound transcription factor analysis}

Complete cDNA and amino acid sequences of transcription factors of the plant species (Table 1) were retrieved from the recently published plant transcription factor database (PlantTFDB v4.0, http://planttfdb.cbi.pku.edu.cn/) (Jin et al. 2017). The consequent sequences were used to perform in silico analysis of membrane-bound proteins via the following bioinformatics tools.

\section{Selection of bioinformatics methods for membrane-bound protein prediction}

Seven prediction methods including TMHMM 2.0 (Krogh et al. 2001), S-TMHMM (Viklund and Elofsson 2004), HMMTOP (Tusnady and Simon 2001), PHOBIUS (Kall et al. 2004), SCAMPI-single (Bernsel et al. 2008), MEMSAT 1.0 (Jones et al. 1994) and TOPPRED (Vonheijne 1992) were chosen for TM prediction. For TMHMM 2.0, transmembrane helices and the locations were predicted; the most likely locations of transmembrane motifs were given, and they were included in this study if they had a probability score greater than 0.90 . The other six predictors were employed to predict transmembrane motifs and the consequent results were filtered and/or combined by using TOPCONS-single software with the default parameters (http://single.topcons.net/) (Hennerdal and Elofsson 2011).

\section{In silico analysis of alternative splicing of MTFs in Arabidopsis and cotton}

Transcriptome sequences of Arabidopsis thaliana (TAIR10_cds_20101214_updated) were downloaded from TAIR (The Arabidopsis Information Resource) website (https://www.arabidopsis.org/). Transcriptome sequences of cotton (G. raimondii) were retrieved from Phytozome (https://phytozome.jgi.doe.gov/pz/portal.htm/\#). 


\section{Manuscript to be reviewed}

115 Results \& Discussion

116 Establishment of workflow for identification of MTFs

117 To perform genome wide analyses of the MTFs in the different plant species, seven tools 118 that are widely used to predict transmembrane segments were selected in present study: 119 TMHMM 2.0 (Krogh et al. 2001), HMMTOP (Tusnady and Simon 2001), PHOBIUS (Kall et 120 al. 2004), S-TMHMM (Viklund and Elofsson 2004), TOPPRED (Vonheijne 1992), 121 SCAMPI-single (Bernsel et al. 2008) and MEMSAT 1.0 (Jones et al. 1994). Their methods 122 are based on different algorithms such as the hidden Markov model (HMM), the artificial 123 neural network (ANN) and hydrophobicity (Table 2). These methods were selected 124 because of their frequent usage in the research community and good results obtained in 125 various studies (Schwacke et al. 2003; Zhang et al. 2015). Moreover, multiple predictors were employed to identify as many reliable membrane proteins as possible. .

Sequences of Arabidopsis transcription factors were extracted from the recently published Plant Transcription Factor Database (PlantTFDB 4.0) (Jin et al. 2017), and they were then subjected to transmembrane region screening using the above predictors. In $A$.

130 thaliana, for example, the genome was predicted to contain 37 to 60 membrane-bound 131 transcription factors (MTFs) depending on the methods used (Fig. 1a). Regardless of the 132 prediction method, most of the resulting MTFs have a single TM span, ranging from $63 \%$ 133 to $93 \%$ (Fig. 1a). The numbers of both predicted MTFs and predicted TM motifs per 134 protein varied among the different methods. This is because the algorithms of the 135 methods are different; some methods may overlook membrane regions that are recognized by other methods (Schwacke et al. 2003). Therefore, a consensus decision

137 was made for topology predictions based on different prediction methods, which could generate data with high accuracy and confidence (Hennerdal and Elofsson 2011). The

139 workflow for MTF identification in the present study is shown in Fig. 1b; plant transcription 140 factor databases were subjected to analysis using the seven bioinformatics tools, 141 including TMHMM 2.0, HMMTOP, PHOBIUS, S-TMHMM, TOPPRED, SCAMPI-single 142 and MEMSAT 1.0. The TOPCONS-single algorithm (Hennerdal and Elofsson 2011) was 


\section{Manuscript to be reviewed}

143 employed to obtain consensus prediction data for membrane proteins predicted by all of

144 the methods except TMHMM 2.0, and the results were then combined with the data

145 predicted by TMHMM 2.0 to constitute the membrane transcription factor database with

146 high confidence (Fig. 1b). The results generated by TMHMM 2.0 were separately

147 analyzed because TMMHM 2.0 is the most accurate software currently available for

148 membrane protein prediction, as it is characterized by a low false-positive rate but a high

149 false-negative rate (Schwacke et al. 2003).

150 We employed this established workflow to identify membrane-bound members from

151 the Arabidopsis transcription factors. The Arabidopsis genome was found to contain 64

152 MTFs (Fig. 1c), among which the number of proteins containing one, two and three

153 membrane segments was 52, 11 and one, respectively. These proteins are classified as

154 belonging to 24 gene families, including 17 NAC members and 5 bZIP members (Fig. 1d).

155 The total number of MTFs identified in the present study was less than that in the 156 previously predicted MTF database (85) in Arabidopsis (Kim et al. 2010), and 43 members 157 overlapped between the two studies, which accounted for $67 \%$ of the MTFs in the present 158 study (Fig. 1e). In contrast to the in silico analysis of Arabidopsis MTFs, this study 159 identified 85 MTFs in the rice (Oryza sativa) genome database (Table 1), which is much 160 more than the 45 members identified in previous research (Kim et al. 2010). The 161 difference between the two studies may be due to the different algorithms used, which is 162 common in the research on membrane protein prediction (Schwacke et al. 2003). 163 However, 85 MTFs were identified in rice in the present study, while only 45 MTFs were 164 identified in rice by Kim et al. 2010 (Table 1). Both the previous (Kim et al. 2010) and the 165 present study have tried to identify all possible MTFs for the scientific community. One 166 major advantage of the workflow in the present study is that these methods are freely 167 available to the scientific community and are applicable to more plant species. The 168 Arabidopsis MTFs identified in this study are highly reliable, because all of the Arabidopsis 169 MTFs functionally elucidated in previous research (Table S1) were present in this study 170 except for a PHD transcription factor (AT5G35210) that was not deposited in PlantTFDB 


\section{Manuscript to be reviewed}

1714.0 (Sun et al. 2011). These results further indicated that the workflow for MTFs

172 identification established in this study can identify MTF with considerable reliability.

173

174

175

176

177

178

179

180

181

182

183

184

185

186

187

188

189

190

191

192

193

194

195

196

197

198

\section{Genome-wide in silico identification of MTFs in plant species}

Genome-wide scale screening of MTFs has been performed in only two plant species: Arabidopsis thaliana and rice (Oryza sativa) (Kim et al. 2010; Seo 2014). To considerably broaden this dataset and cover other plant species, we performed extensive genome-wide in silico analyses of various plant species as follows (Table 1). First, the sequences of plant transcription factors ranging from 230 to 3714 loci were retrieved for different species from PlantTFDB 4.0 (Jin et al. 2017). Second, the transcription factor databases were screened for transmembrane motifs. Finally, a highly reliable database has been established that consists of 1089 MTFs (Table 1). The number of MTFs in each species varied from 37 to 117 and accounted for approximately $3 \%$ to $16 \%$ of the total transcription factors. Relevant information about the MTFs, including the gene loci, transmembrane region and gene family, are shown in Table S2.

\section{Gene family classification of MTFs in plant species}

The 1089 MTFs identified in 14 plant species in this study belong to 52 families (Table 1). Twenty-eight additional gene families of MTFs were identified from plant species besides A. thaliana (At) (Table 3). A total of 26 families containing MTFs were identified from more than seven plant species including the AP2, B3, bHLH, bZIP, C2H2, C3H, Dof, ERF, FAR1, G2-like, GATA, GRAS, HB-other, HD-ZIP, LBD, MIKC, M-type, MYB, MYB-related, NAC, NF-X1, Nin-like, S1Fa-like, SBP, Trihelix and WRKY families (Table 3). Moreover, nine gene families of MTFs, including bHLH, C2H2, G2-like, NAC, C3H, ERF, HB-other, M-type and NF-X1, were identified from more than 12 plant species. Notably, the bZIP, MYB-related, S1Fa-like and SBP families of MTFs were identified from all 14 of the plant species. In contrast, several gene families of MTFs were identified from only a few plant species (no more than two), namely, the BBR-BPC, EIL, GeBP, GRF, RAV, SAP and Whirly families. We further summarized the total number of MTFs in the 14 species, and the top six families with the highest numbers of MTFs were as follows: NAC (128), SBP 


\section{Manuscript to be reviewed}

199 (77), C2H2 (70), MYB-related (67) and bHLH (65) (Fig. S1). These results showed that

200 MTFs are highly diverse in various plant species. Considering that only a limited number 201 MTFs (Seo 2014), e.g., eight NAC and three bZIP transcription factors in Arabidopsis, 202 have been functionally studied, the other MTFs deserve much more attention in further 203 research due to the important role of MTFs in gene expression.

204 Analysis of transmembrane motifs of MTFs in plant species

205 Among the 1089 MTFs identified from 14 plant species, the number of TM motifs varied 206 from one to 13. Most MTFs in the plant species, except for those in C. reinhardtii, have 207 one or two TM motifs (Fig. 2a). We summarized the total number of TM regions of the 208 MTFs in all plant species; proteins containing one and two TM motifs accounted for $71.5 \%$, 209 and $21.1 \%$ of the MTFs, respectively.

The location patterns of TM motifs within protein sequences were analyzed, and the major types of TM motif locations were shown in Fig. 2b. Among the MTFs containing one 212 TM region, a total of $311(39.9 \%), 360(46.2 \%)$, and $108(13.9 \%)$ proteins had their TM motifs in the N-terminal, C-terminal and central region of their sequences, respectively.

214 This was slightly different from a previous study on the MTFs of $A$. thaliana, in which more 215 than 70\% members had TM motifs in their C-terminal regions (Kim et al. 2010). For the 216230 MTF members containing two TM motifs, a total of $51(22.2 \%)$ and $46(20 \%)$ 217 members had their TM regions in the $\mathrm{N}$-terminal and $\mathrm{C}$-terminal regions, respectively; an 218 additional 31 MTF members (13.5\%) had one TM motif located in the $\mathrm{N}$-terminal region 219 and the other motif located in the C-terminal region (Fig. 2b). Taken together, most MTFs 220 in plant species have one or two TM domains, which are located in either their $\mathrm{N}$ - or 221 C-terminal region. These results indicated that proteolytic cleavage is a conserved 222 mechanism for MTFs in various plant species, as it is likely to release an intact functional 223 transcription factor since the MTF was cleaved by protease near the site of the 224 transmembrane domain. Such proteolytic process-dependent activation has been well 225 established in Arabidopsis MTFs (Seo 2014). Furthermore, the variance in TM motifs in 


\section{Manuscript to be reviewed}

226 MTFs may lead to slight differences in the details of the mechanism of MTF activation by 227 proteolytic processing.

228

229

230

231

232

233

234

235

236

237

238

239

240

241

242

243

244

245

246

247

248

249

250

251

252

253

\section{Possible regulation of MTFs by alternative splicing}

MTFs were once considered to be activated solely by proteolytic processing. Interestingly, recent research concerning ZmbZIP60 and OsbZIP74 suggested that alternative splicing (ALS) is also responsible for the activation of MTFs (Li et al. 2012; Lu et al. 2012). To test whether ALS-assisted activation of MTFs was also employed by other plants' MTFs, MTF databases of Arabidopsis and cotton (G. raimondii) were selected, and in silico analyses were performed. The results are shown in Fig. 3. In an Arabidopsis MTF member (AT5G1050.3), for example, two splice variants (AT5G1050.1 and AT5G1050.2) encode proteins that maintain the nuclear transcription factor domain while deleting the TM region (Fig. 3a and b). Thus, ALS was proposed to be another mechanism for the activation of MTFs, in which the splice variant without the sequence encoding a TM motif would be transcribed under specific physiological conditions. In this case, the nuclear transcription factors translocate into the nucleus and become active immediately since they were translated in the cytoplasm. The regulation of MTFs by ALS may be important in the late stage of plants in response to various stimuli. Interestingly, approximately 30\% (19/64) and $53 \%$ (59/111) of genes encoding MTFs in Arabidopsis and cotton, respectively, were also shown to generate alternative splicing variants coding for proteins that only maintain the transcription factor domain with nuclear localization signal while deleting the TM motif (Fig. 3c \& d and Table S3). Hence, these MTFs could be regulated by ALS in Arabidopsis and cotton. Moreover, ALS seems to be involved in the activation of a wide range of MTF families, such as the NAC, bHLH and MYB-related families, in Arabidopsis and cotton (Table S3). Thus, an ALS-dependent activation mechanism may also be employed by the MTFs of other plant species. However, a further "wet experiment" is needed to verify this hypothesis.

Conclusion 


\section{Manuscript to be reviewed}

254 The present study performed a systematic analysis of MTFs in plant lineages at the 255 genome level. A total of 1089 MTFs belonging to 52 gene families were identified in 14 256 plant species. Most of the MTFs have TM domains located in either the $\mathrm{N}$ - or C-terminal 257 regions, indicating proteolytic cleavage could be a conserved mechanism of MTF 258 activation in plant species. Additionally, approximately half of the MTFs in the genome of 259 either Arabidopsis or cotton can potentially be regulated by ALS, indicating that ALS may 260 be another conserved activation mechanism for MTFs. The present study provides 261 invaluable information for the research community.

262

263

\section{References:}

264 Bernsel A, Viklund H, Falk J, Lindahl E, von Heijne G, Elofsson A (2008) Prediction of 265 membrane-protein topology from first principles. P NATL ACAD SCI USA 105: 7177-7181

266 Che P, Bussell JD, Zhou W, Estavillo GM, Pogson BJ, Smith SM (2010) Signaling from the 267 endoplasmic reticulum activates brassinosteroid signaling and promotes acclimation to stress 268 in Arabidopsis. SCI SIGNAL 3: ra69.

269 De Clercq I, Vermeirssen V, Van Aken O, Vandepoele K, Murcha MW, Law SR, Inze A, Ng S, 270 Ivanova A, Rombaut D, van de Cotte B, Jaspers P, Van de Peer Y, Kangasjarvi J, Whelan J, 271 Van Breusegem $F$ (2013) The membrane-bound NAC transcription factor ANAC013 functions 272 in mitochondrial retrograde regulation of the oxidative stress response in Arabidopsis. PLANT 273 CELL 25: 3472-3490

274 Gao H, Brandizzi F, Benning C, Larkin RM (2008) A membrane-tethered transcription factor 275 defines a branch of the heat stress response in Arabidopsis thaliana. P NATL ACAD SCI USA 276 105: 16398-16403

277 Hennerdal A, Elofsson A (2011) Rapid membrane protein topology prediction. 278 BIOINFORMATICS 27: 1322-1323

279 Iwata Y, Fedoroff NV, Koizumi N (2008) Arabidopsis bZIP60 is a proteolysis-activated 280 transcription factor involved in the endoplasmic reticulum stress response. PLANT CELL 20: $281 \quad 3107-3121$

282 Iwata $\mathrm{Y}$, Koizumi N (2005) An Arabidopsis transcription factor, AtbZIP60, regulates the 283 endoplasmic reticulum stress response in a manner unique to plants. P NATL ACAD SCI USA 284 102: 5280-5285

285 Jin J, Tian F, Yang DC, Meng YQ, Kong L, Luo J, Gao G (2016) PlantTFDB 4.0: toward a 286 central hub for transcription factors and regulatory interactions in plants. NUCLEIC ACIDS 287 RES

288 Jones DT, Taylor WR, Thorton JM (1994) A model recognition approach to the prediction of 289 all-helical membrane-protein structure and topology. BIOCHEMISTRY-US 33: 3038-3049

290 Kall L, Krogh A, Sonnhammer E (2004) A combined transmembrane topology and signal 291 peptide prediction method. J MOL BIOL 338: 1027-1036 


\section{Manuscript to be reviewed}

292 Kim MJ, Park M, Seo PJ, Song J, Kim H, Park C (2012) Controlled nuclear import of the

293 transcription factor NTL6 reveals a cytoplasmic role of SnRK2.8 in the drought-stress 294 response. BIOCHEM J 448: 353-363

295 Kim S, Lee A, Yoon H, Park C (2008) A membrane-bound NAC transcription factor NTL8

296 regulates gibberellic acid-mediated salt signaling in Arabidopsis seed germination. PLANT J

297 55: 77-88

298 Kim S, Lee S, Seo PJ, Kim S, Kim J, Park C (2010) Genome-scale screening and molecular

299 characterization of membrane-bound transcription factors in Arabidopsis and rice. GENOMICS

300 95: 56-65

301 Kim Y, Kim S, Park J, Park H, Lim M, Chua N, Park C (2006) A membrane-bound NAC

302 transcription factor regulates cell division in Arabidopsis. PLANT CELL 18: 3132-3144

303 Krogh A, Larsson B, von Heijne G, Sonnhammer E (2001) Predicting transmembrane protein

304 topology with a hidden Markov model: Application to complete genomes. J MOL BIOL 305:

$305 \quad 567-580$

306 Lee S, Seo PJ, Lee H, Park C (2012) A NAC transcription factor NTL4 promotes reactive

307 oxygen species production during drought-induced leaf senescence in Arabidopsis. PLANT J

308 70: 831-844

309 Li P, Wind JJ, Shi X, Zhang H, Hanson J, Smeekens SC, Teng S (2011) Fructose sensitivity is

310 suppressed in Arabidopsis by the transcription factor ANAC089 lacking the membrane-bound 311 domain. P NATL ACAD SCI USA 108: 3436-3441

$312 \mathrm{Li}$ Y, Humbert S, Howell SH (2012) ZmbZIP60 mRNA is spliced in maize in response to ER 313 stress. BMC research notes 5: 144

314 Lu S, Yang Z, Sun L, Sun L, Song Z, Liu J (2012) Conservation of IRE1-regulated bZIP74

315 mRNA unconventional splicing in rice (Oryza sativa L.) involved in ER stress responses. MOL

316 PLANT 5: 504-514

$317 \mathrm{Ng} \mathrm{S}$, Ivanova A, Duncan O, Law SR, Van Aken O, De Clercq I, Wang Y, Carrie C, Xu L,

318 Kmiec B, Walker H, Van Breusegem F, Whelan J, Giraud E (2013) A membrane-bound NAC

319 transcription factor, ANAC017, mediates mitochondrial retrograde signaling in Arabidopsis.

320 PLANT CELL 25: 3450-3471

321 Park J, Kim Y, Kim S, Jung J, Woo J, Park C (2011) Integration of auxin and salt signals by the

322 NAC transcription factor NTM2 during seed germination in Arabidopsis. PLANT PHYSIOL 156:

$323 \quad 537-549$

324 Schwacke R, Schneider A, van der Graaff E, Fischer K, Catoni E, Desimone M, Frommer WB,

325 Flugge UI, Kunze R (2003) ARAMEMNON, a novel database for Arabidopsis integral

326 membrane proteins. PLANT PHYSIOL 131: 16-26

327 Seo PJ (2014) Recent advances in plant membrane-bound transcription factor research:

328 Emphasis on intracellular movement. J INTEGR PLANT BIOL 56: 334-342

329 Seo PJ, Kim MJ, Park J, Kim S, Jeon J, Lee Y, Kim J, Park C (2010) Cold activation of a

330 plasma membrane-tethered NAC transcription factor induces a pathogen resistance response

331 in Arabidopsis. PLANT J 61: 661-671

332 Seo PJ, Kim S, Park C (2008) Membrane-bound transcription factors in plants. TRENDS

333 PLANT SCI 13: 550-556 
334 Slabaugh E, Held M, Brandizzi F (2011) Control of root hair development in Arabidopsis

335 thaliana by an endoplasmic reticulum anchored member of the R2R3-MYB transcription factor

336 family. PLANT J 67: 395-405

337 Sun X, Feng P, Xu X, Guo H, Ma J, Chi W, Lin R, Lu C, Zhang L (2011) A chloroplast

338 envelope-bound PHD transcription factor mediates chloroplast signals to the nucleus. NAT

339 COMMUN 2:477.

340 Tusnady GE, Simon I (2001) The HMMTOP transmembrane topology prediction server.

341 BIOINFORMATICS 17: 849-850

342 Viklund $\mathrm{H}$, Elofsson A (2004) Best alpha-helical transmembrane protein topology predictions

343 are achieved using hidden Markov models and evolutionary information. PROTEIN SCI 13:

$344 \quad 1908-1917$

345 Vonheijne G (1992) Membrane-protein structure prediction-hydrophobicity analysis and the

346 positive-inside rule. J MOL BIOL 225: 487-494

347 Yang Z, Lu S, Wang M, Bi D, Sun L, Zhou S, Song Z, Liu J (2014) A plasma

348 membrane-tethered transcription factor, NAC062/ANAC062/NTL6, mediates the unfolded

349 protein response in Arabidopsis. PLANT J 79: 1033-1043

350 Yoon H, Kim S, Kim S, Park C (2008) Regulation of leaf senescence by NTL9-mediated

351 osmotic stress signaling in Arabidopsis. MOL CELLS 25: 438-445

352 Zhang Y, Li Y, Zhang Y, Wang Z, Zhao M, Su N, Zhang T, Chen L, Wei W, Luo J, Zhou Y, Xu

$353 \mathrm{Y}, \mathrm{Xu} \mathrm{Y}, \mathrm{Xu} \mathrm{P}, \mathrm{Li} \mathrm{W}, \mathrm{Tao} \mathrm{Y}$ (2015) Quantitative proteomics reveals membrane

354 protein-mediated

355 hypersaline sensitivity and adaptation in halophilic Nocardiopsis xinjiangensis. J PROTEOME

356 RES 15: 68-85

357

358 


\section{Manuscript to be reviewed}

(a)

a)

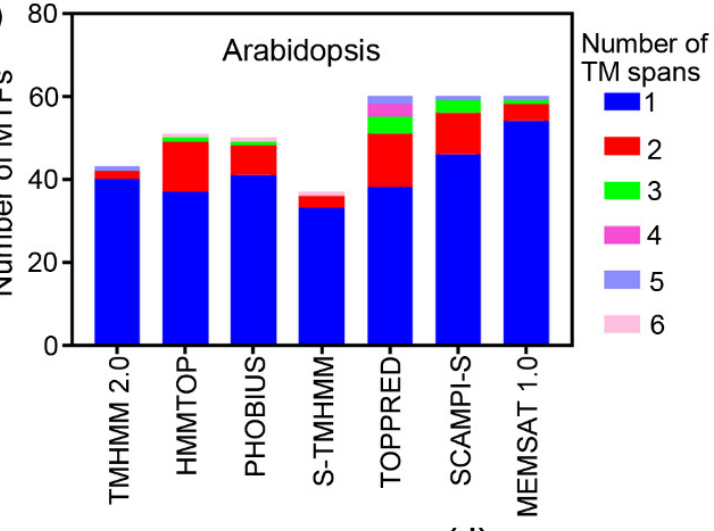

(c)

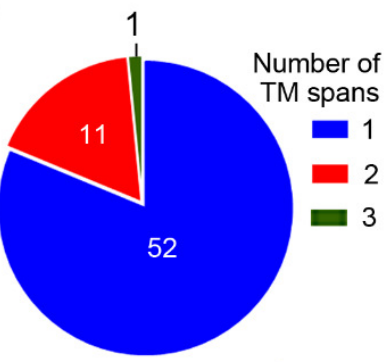

(d)

(b)

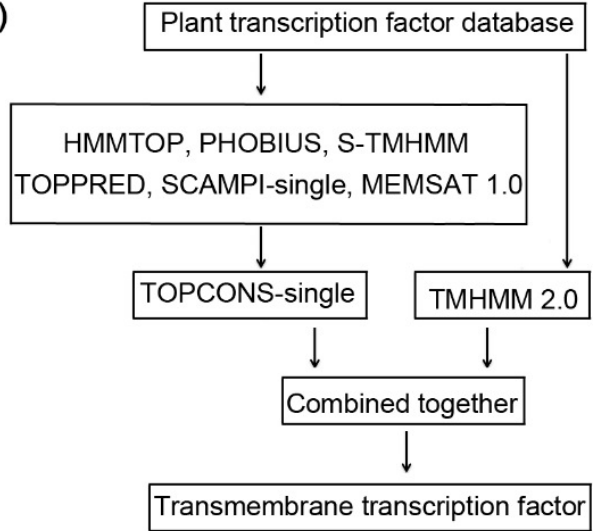

359

(e)

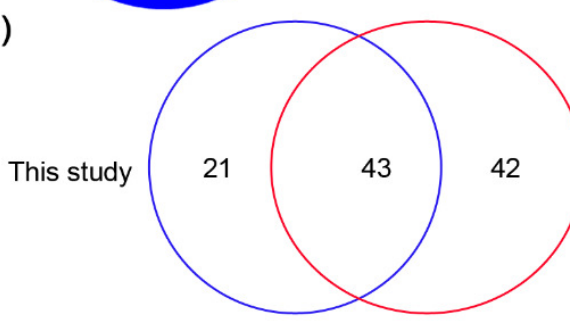

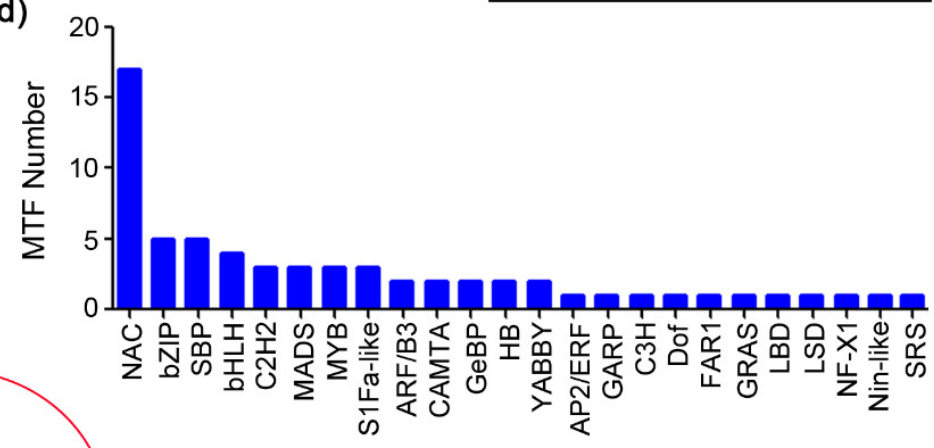

Arabidopsis gene family that contains MTFs Kim et al. 2010

Fig. 1. Workflow for identification of membrane-bound transcription factors in plant

361 species. (a) Prediction of transmembrane (TM) spans of Arabidopsis by different methods; (b) workflow for identification of MTFs in plant species; (c) Arabidopsis MTFs identified in present study; (d) gene family of Arabidopsis MTFs; (e) Comparison of the Arabidopsis MTFs identified in this study with the previous research.

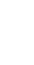




\section{Manuscript to be reviewed}

(a)

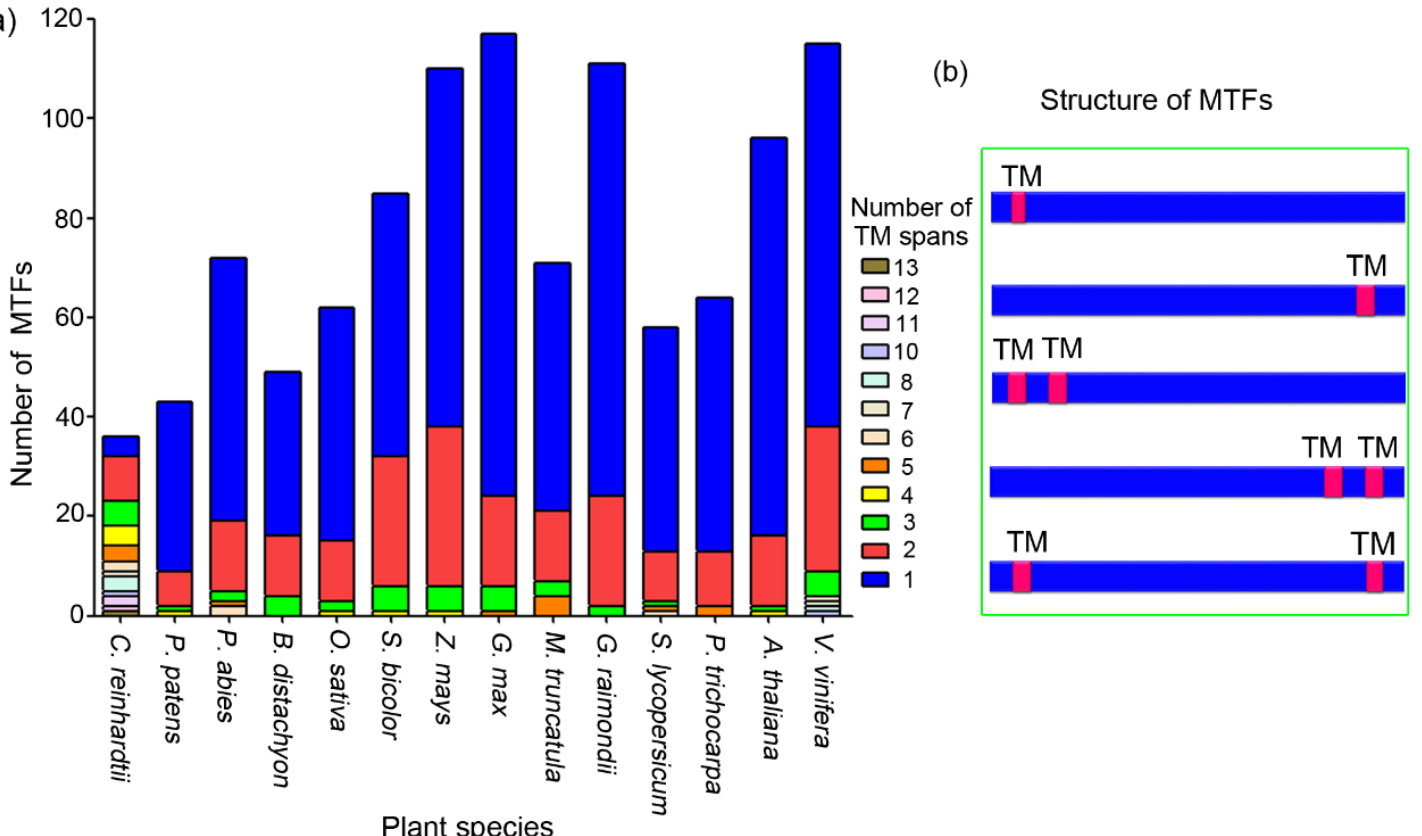

370

371

372

373

374

375

376

377

378

379

380

381

382

383

384

Fig. 2. Analysis of transmembrane motifs of membrane-bound transcription factors in plant species. (a) Number of TM spans in MTFs; (b) typical locations of TM spans in MTFs. TM, transmembrane; MTF, membrane-bound transcription factor.

5

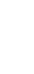


(a)

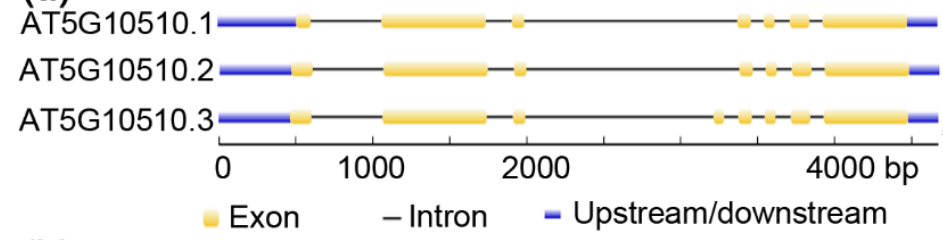

(b)

AT5G10510.1

AT5G10510.2

AT5G10510.3

(c)

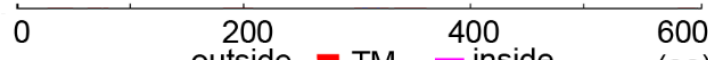
- outside $=$ TM - inside (aa)

Cotton
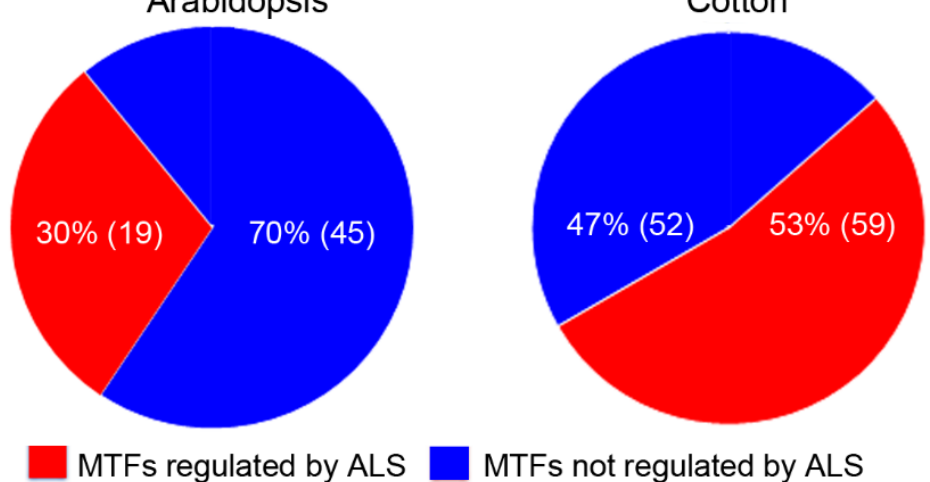

MTFs not regulated by ALS

386 Fig. 3. Alternative splicing of membrane-bound transcription factors in Arabidopsis (Arabidopsis thaliana) and cotton (Gossypium raimondii). (a) Arabidopsis membrane-bound transcription factor (AT5G10510) undergoes alternative spicing (ALS); (b) Alternatively spliced forms of Arabidopsis AT5G10510 lack a TM domain; (c) Potential ALS dependent activation of membrane-bound transcription factors in genome of 
Table 1. Genome information and sizes of the MTFs of analyzed plant species.

\begin{tabular}{|c|c|c|c|c|c|}
\hline Species & Common name & $\begin{array}{l}\text { Genome } \\
\text { size (Mbp) }\end{array}$ & $\begin{array}{l}\text { Chromosome } \\
(1 \mathrm{~N})\end{array}$ & TF loci & $\begin{array}{l}\text { MTF } \\
\text { loci }\end{array}$ \\
\hline \multicolumn{6}{|l|}{ Chlorophyta } \\
\hline $\begin{array}{l}\text { Chlamydomonas } \\
\text { reinhardtii }\end{array}$ & Green algae & 112 & 17 & 230 & 37 \\
\hline \multicolumn{6}{|l|}{ Bryophyta } \\
\hline Physcomitrella patens & Moss & 480 & 27 & 1079 & 49 \\
\hline \multicolumn{6}{|l|}{ Gymnosperm } \\
\hline Picea abies & Norway spruce & $19.6 \mathrm{~Gb}$ & 12 & 1851 & 71 \\
\hline \multicolumn{6}{|l|}{ Monocot } \\
\hline Brachypodium distachyon & Brachypodium & 272 & 5 & 1557 & 50 \\
\hline Oryza sativa & Japanese rice & 372 & 12 & 1859 & 85 \\
\hline Sorghum bicolor & Sorghum & 730 & 10 & 1826 & 62 \\
\hline Zea mays & Maize & 3,000 & 10 & 2231 & 110 \\
\hline \multicolumn{6}{|l|}{ Eudicot } \\
\hline Glycine max & Soybean & 979 & 20 & 3714 & 117 \\
\hline Medicago truncatula & Barrel medic & 258 & 8 & 1577 & 71 \\
\hline Gossypium raimondii & Cotton & 880 & 13 & 2634 & 111 \\
\hline Solanum lycopersicum & Tomato & 900 & 12 & 1845 & 58 \\
\hline Populus trichocarpa & $\begin{array}{l}\text { Western balsam } \\
\text { poplar }\end{array}$ & 423 & 19 & 2455 & 89 \\
\hline Arabidopsis thaliana & Thale cress & 135 & 5 & 1716 & 64 \\
\hline Vitis vinifera & Wine grape & 487 & 19 & 1276 & 115 \\
\hline
\end{tabular}




\begin{tabular}{llll}
\hline Topology predictor & Algorithm & Reference & Tab \\
TMHMM 2.0 & HMM & le 2. \\
HMMTOP & HMM & (Tusnady \& Simon, 2001) & The \\
PHOBIUS & HMM & (Kall et al., 2004) & me \\
S-TMHMM & HMM & (Viklund and Elofsson, 2004) & ane \\
TOPPRED & Hydrophobicity & (von Heijne, 1992) & topo \\
& profiles & & logy \\
SCAMPI-single & Hydrophobicity+Model & (Bernsel et al., 2008) & pred \\
MEMSAT 1.0 & ANN+Grammer & (Jones et al., 1994) & ictio \\
$n$ & &
\end{tabular}


Table 3. Membrane-bound transcription factors (MTFs) in gene families of plant species.

\begin{tabular}{|c|c|c|c|c|c|c|c|c|c|c|c|c|c|c|}
\hline \multicolumn{3}{|c|}{ Family } & \multicolumn{12}{|c|}{ Plant species (MTF number) } \\
\hline \multicolumn{2}{|c|}{ Chlorophyte } & \multirow{2}{*}{$\begin{array}{c}\begin{array}{c}\text { Bryo- } \\
\text { phyte }\end{array} \\
\text { Pp }\end{array}$} & \multirow{2}{*}{$\begin{array}{c}\text { Gymno- } \\
\text { sperm }\end{array}$} & \multicolumn{4}{|c|}{ Monocot } & \multicolumn{7}{|c|}{ Eudicot } \\
\hline & $\mathrm{Cr}$ & & & $\mathrm{Bd}$ & Os & $\mathrm{Sb}$ & $\mathrm{Zm}$ & $\mathrm{Gm}$ & Mt & $\mathrm{Gr}$ & SI & $\mathrm{Pt}$ & At & Vv \\
\hline AP2 & 4 & 0 & 0 & 1 & 1 & 0 & 0 & 1 & 1 & 0 & 0 & 0 & 1 & 2 \\
\hline ARF & 0 & 0 & 3 & 1 & 0 & 0 & 2 & 2 & 0 & 2 & 0 & 1 & 0 & 0 \\
\hline ARR-B & 0 & 0 & 0 & 0 & 0 & 0 & 0 & 1 & 0 & 1 & 0 & 1 & 0 & 1 \\
\hline B3 & 0 & 4 & 0 & 3 & 2 & 1 & 0 & 1 & 2 & 1 & 0 & 0 & 2 & 2 \\
\hline $\begin{array}{l}\text { BBR-BP } \\
\text { C }\end{array}$ & 0 & 0 & 1 & 0 & 0 & 0 & 0 & 0 & 0 & 0 & 0 & 1 & 0 & 0 \\
\hline BES1 & 0 & 1 & 1 & 0 & 0 & 0 & 1 & 1 & 0 & 1 & 0 & 1 & 0 & 0 \\
\hline bHLH & 4 & 2 & 3 & 3 & 6 & 5 & 8 & 13 & 3 & 4 & 0 & 4 & 4 & 4 \\
\hline bZIP & 3 & 6 & 5 & 3 & 4 & 4 & 6 & 5 & 3 & 5 & 5 & 9 & 5 & 4 \\
\hline $\mathrm{C} 2 \mathrm{H} 2$ & 0 & 3 & 4 & 4 & 5 & 6 & 5 & 9 & 6 & 11 & 5 & 3 & 3 & 6 \\
\hline $\mathrm{C} 3 \mathrm{H}$ & 3 & 2 & 7 & 3 & 3 & 0 & 2 & 2 & 3 & 2 & 0 & 5 & 1 & 5 \\
\hline CAMTA & 0 & 0 & 0 & 0 & 0 & 0 & 2 & 2 & 2 & 5 & 0 & 0 & 2 & 0 \\
\hline CO-like & 1 & 0 & 0 & 0 & 0 & 0 & 1 & 0 & 0 & 0 & 0 & 2 & 0 & 0 \\
\hline CPP & 1 & 0 & 0 & 0 & 0 & 0 & 0 & 0 & 0 & 0 & 1 & 1 & 0 & 1 \\
\hline DBB & 0 & 0 & 0 & 0 & 0 & 0 & 1 & 2 & 0 & 0 & 0 & 2 & 0 & 0 \\
\hline Dof & 0 & 0 & 2 & 1 & 1 & 0 & 1 & 1 & 0 & 1 & 0 & 0 & 1 & 4 \\
\hline E2F/DP & 0 & 0 & 1 & 0 & 0 & 0 & 0 & 2 & 0 & 1 & 0 & 1 & 0 & 0 \\
\hline EIL & 0 & 0 & 0 & 0 & 1 & 0 & 0 & 0 & 1 & 0 & 0 & 0 & 0 & 0 \\
\hline ERF & 0 & 3 & 3 & 1 & 3 & 3 & 7 & 1 & 1 & 3 & 2 & 1 & 0 & 10 \\
\hline FAR1 & 0 & 0 & 0 & 3 & 11 & 2 & 3 & 0 & 10 & 3 & 0 & 2 & 1 & 3 \\
\hline G2-like & 1 & 2 & 1 & 2 & 4 & 0 & 3 & 2 & 1 & 4 & 3 & 1 & 1 & 2 \\
\hline GATA & 2 & 1 & 0 & 1 & 2 & 1 & 1 & 2 & 2 & 0 & 0 & 1 & 0 & 2 \\
\hline GeBP & 0 & 0 & 0 & 0 & 0 & 1 & 0 & 0 & 0 & 0 & 0 & 0 & 2 & 0 \\
\hline GRAS & 0 & 0 & 3 & 1 & 4 & 11 & 6 & 0 & 1 & 0 & 2 & 0 & 1 & 4 \\
\hline GRF & 0 & 0 & 0 & 0 & 0 & 0 & 0 & 1 & 0 & 1 & 0 & 0 & 0 & 0 \\
\hline $\begin{array}{l}\text { HB-othe } \\
r\end{array}$ & 1 & 0 & 3 & 1 & 2 & 0 & 4 & 3 & 1 & 4 & 3 & 5 & 2 & 4 \\
\hline HD-ZIP & 0 & 1 & 0 & 1 & 0 & 1 & 6 & 3 & 0 & 6 & 0 & 1 & 0 & 3 \\
\hline HSF & 0 & 0 & 1 & 2 & 0 & 1 & 1 & 0 & 0 & 0 & 0 & 0 & 0 & 1 \\
\hline LBD & 0 & 2 & 1 & 0 & 0 & 0 & 0 & 0 & 1 & 1 & 1 & 0 & 1 & 1 \\
\hline LSD & 0 & 0 & 0 & 0 & 2 & 0 & 0 & 1 & 0 & 0 & 0 & 1 & 1 & 1 \\
\hline MIKC & 0 & 0 & 0 & 2 & 1 & 2 & 3 & 2 & 0 & 4 & 0 & 1 & 1 & 2 \\
\hline M-type & 0 & 1 & 3 & 1 & 3 & 1 & 2 & 4 & 2 & 3 & 7 & 3 & 2 & 0 \\
\hline MYB & 1 & 1 & 0 & 0 & 1 & 2 & 1 & 2 & 0 & 4 & 0 & 4 & 1 & 11 \\
\hline $\begin{array}{l}\text { MYB-rel } \\
\text { ated }\end{array}$ & 4 & 1 & 6 & 2 & 4 & 2 & 10 & 7 & 6 & 6 & 5 & 8 & 2 & 2 \\
\hline
\end{tabular}


Continued-Table 3. Membrane-bound transcription factors (MTFs) in gene families of plant species.

\begin{tabular}{|c|c|c|c|c|c|c|c|c|c|c|c|c|c|c|}
\hline \multicolumn{3}{|c|}{ Family } & \multicolumn{12}{|c|}{ Plant species (MTF number) } \\
\hline \multicolumn{2}{|c|}{$\begin{array}{r}\text { Chlorophyt } \\
\mathrm{e}\end{array}$} & \multirow{2}{*}{$\begin{array}{c}\begin{array}{c}\text { Bryop-h } \\
\text { yte }\end{array} \\
\mathrm{Pp}\end{array}$} & \multirow{2}{*}{$\begin{array}{c}\begin{array}{c}\text { Gymno } \\
\text {-sperm }\end{array} \\
\mathrm{Pa}\end{array}$} & \multicolumn{4}{|c|}{ Monocot } & \multicolumn{7}{|c|}{ Eudicot } \\
\hline & $\mathrm{Cr}$ & & & $\mathrm{Bd}$ & Os & $\mathrm{Sb}$ & $\mathrm{Zm}$ & $\begin{array}{l}\mathrm{G} \\
\mathrm{m}\end{array}$ & Mt & $\mathrm{Gr}$ & SI & $\mathrm{Pt}$ & At & Vv \\
\hline NAC & 0 & 6 & 7 & 7 & 6 & 7 & 11 & 14 & 5 & 15 & 11 & 12 & 17 & 10 \\
\hline $\begin{array}{l}\text { NF-X } \\
1\end{array}$ & 1 & 1 & 0 & 0 & 1 & 1 & 1 & 2 & 1 & 1 & 1 & 1 & 1 & 2 \\
\hline $\begin{array}{l}\text { NF-Y } \\
A\end{array}$ & 0 & 0 & 1 & 0 & 1 & 0 & 1 & 1 & 1 & 0 & 0 & 0 & 0 & 0 \\
\hline $\begin{array}{l}\text { NF-Y } \\
B\end{array}$ & 0 & 0 & 1 & 0 & 0 & 0 & 0 & 3 & 0 & 1 & 2 & 0 & 0 & 1 \\
\hline $\begin{array}{l}\text { NF-Y } \\
\text { C }\end{array}$ & 0 & 1 & 1 & 1 & 1 & 0 & 0 & 0 & 1 & 0 & 1 & 0 & 0 & 0 \\
\hline $\begin{array}{l}\text { Nin-li } \\
\text { ke }\end{array}$ & 1 & 2 & 0 & 1 & 1 & 0 & 0 & 2 & 1 & 1 & 0 & 0 & 1 & 2 \\
\hline RAV & 0 & 0 & 0 & 0 & 0 & 0 & 0 & 0 & 0 & 0 & 0 & 2 & 0 & 0 \\
\hline $\begin{array}{l}\text { S1Fa } \\
\text {-like }\end{array}$ & 1 & 2 & 2 & 1 & 2 & 1 & 2 & 4 & 3 & 4 & 1 & 2 & 3 & 2 \\
\hline SAP & 0 & 0 & 0 & 0 & 0 & 0 & 0 & 0 & 1 & 1 & 0 & 0 & 0 & 0 \\
\hline SBP & 9 & 4 & 2 & 4 & 5 & 5 & 7 & 9 & 3 & 9 & 3 & 8 & 5 & 4 \\
\hline SRS & 0 & 0 & 0 & 0 & 0 & 0 & 0 & 1 & 2 & 1 & 0 & 1 & 1 & 1 \\
\hline TALE & 0 & 0 & 0 & 0 & 0 & 1 & 0 & 0 & 0 & 0 & 1 & 0 & 0 & 1 \\
\hline TCP & 0 & 0 & 2 & 0 & 0 & 0 & 3 & 0 & 0 & 0 & 0 & 1 & 0 & 5 \\
\hline $\begin{array}{l}\text { Trihel } \\
\text { ix }\end{array}$ & 0 & 2 & 2 & 0 & 0 & 0 & 3 & 3 & 0 & 4 & 2 & 1 & 0 & 5 \\
\hline $\begin{array}{l}\text { Whirl } \\
y\end{array}$ & 0 & 0 & 0 & 0 & 0 & 0 & 0 & 0 & 0 & 0 & 0 & 0 & 0 & 1 \\
\hline WOX & 0 & 0 & 0 & 0 & 0 & 0 & 1 & 0 & 0 & 0 & 1 & 0 & 0 & 1 \\
\hline $\begin{array}{l}\text { WRK } \\
\text { Y }\end{array}$ & 0 & 1 & 5 & 0 & 6 & 3 & 3 & 4 & 5 & 1 & 1 & 0 & 0 & 2 \\
\hline $\begin{array}{l}\text { YAB } \\
\text { BY }\end{array}$ & 0 & 0 & 0 & 0 & 0 & 1 & 2 & 3 & 0 & 0 & 0 & 2 & 2 & 0 \\
\hline $\begin{array}{l}\text { ZF-H } \\
D\end{array}$ & 0 & 0 & 0 & 0 & 2 & 0 & 0 & 1 & 2 & 0 & 0 & 0 & 0 & 3 \\
\hline
\end{tabular}

